\title{
The Novel Coronavirus-Implications of a Global Pandemic on the Practice of Ophthalmology
}

\author{
Cole M Milliken, Joshua Van Swol, and Karoline M Rocha \\ Storm Eye Institute, Medical University of South Carolina, Charleston, SC, USA
}

DOI: https://doi.org/10.17925/USOR.2020.13.2.58

$\mathrm{T}$ he year 2020 was dominated by the meteoric rise of severe acute respiratory syndrome coronavirus 2, and coronavirus disease (COVID-19). Multiple lifestyle changes, including work from home orders and social distancing, have become the new normal as the world attempts to grapple with the uncertainty resulting from this pandemic. The impact of COVID-19 has been far reaching, disrupting many facets of daily life for people all across the world. Nearly every industry has been required to modify its practice by adopting stricter safety recommendations. Providing quality healthcare in accordance with these guidelines has proved to be a challenge. Ophthalmology has faced unique barriers resulting from COVID-19 because of the proximity to the patient and the duration of the examination required, the diversity and volume of patient encounters, and the unclear ocular manifestations of the virus. This editorial aims to summarize recent literature pertaining to the epidemiology of COVID-19, ocular manifestations of the virus, and information on continued safe practice.

\section{Keywords}

COVID-19, ophthalmology, telehealth

Disclosures: Cole M Milliken, Joshua Van Swol, and Karoline M Rocha have no financial or non-financial relationships or activities to declare in relation to this article. Review Process: Double-blind peer review.

Compliance with Ethics: This article is an opinion piece and does not report on new clinical data, or any studies with human or animal subjects performed by the author.

Authorship: The named authors meet the Internationa Committee of Medical Journal Editors (ICMJE) criteria for authorship of this manuscript, take responsibility for the integrity of the work as a whole, and have given final approval for the version to be published.

Access: This article is freely accessible at touchOPHTHALMOLOGY.com (c) Touch Medical Media 2021.

Received: November 12, 2020

Accepted: December 15, 2020

Published Online: January 22, 2021

Citation: US Ophthalmic Review. 2020;13(2):58-60

Corresponding author: Cole M Milliken, Storm Eye Institute, Medical University of South Carolina, 167 Ashley Avenue, Charleston, SC 29425, USA.

E: millikec@musc.edu

Support: No funding was received in the publication of this article.

\section{Epidemiology}

According to data collected by the Centers for Disease Control and Prevention (CDC), as of December 2020, there have been over 16 million cases of coronavirus disease (COVID-19) in the USA alone, resulting in over 300,000 reported deaths. Worldwide case numbers stand at a staggering 70 million, with over 1.5 million documented deaths.' ${ }^{1}$ During these uncertain times, hospitals and private practices alike are innovating new methods to keep both employees and patients safe. Ophthalmology finds itself with a unique occupational hazard due to the proximity to patients during examination. ${ }^{2}$ Dr Li Wenliang, an ophthalmologist practicing in China, was one of the first providers to alert Chinese government officials to the dangers of COVID-19 before tragically contracting the illness in January 2020 and succumbing to it on February $7,2020 .{ }^{3}$ It remains paramount that ophthalmologists stay up to date with the most current safety recommendations and heed the appropriate precautionary measures.

\section{Ocular manifestations}

Despite the severe pulmonary manifestations of the novel coronavirus, studies on ocular involvement demonstrate limited disease burden at this time. Research performed in the Hubei Province of China illustrated that approximately one-third of confirmed positive COVID-19 patients show ocular symptoms, which include conjunctivitis, hyperemia, chemosis, epiphora, and increased secretions. ${ }^{4}$ Few case reports in the literature have demonstrated ocular findings, such as conjunctivitis, as the presenting symptom(s) of COVID-19, causing concern due to the non-specific nature of the presentation. ${ }^{5}$ In line with the most recent reviews, there have been no reports of severe ophthalmic manifestations, such as corneal scarring, viral keratitis, or conjunctivitis, resulting in pseudo-membrane formation associated with COVID-19 infection. ${ }^{6}$ More visually threatening conditions, including retinitis, anterior uveitis, and optic neuritis, have been observed in animal models,

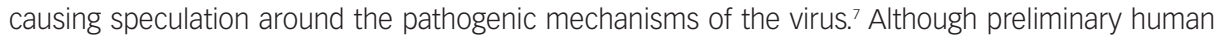
studies do not demonstrate severe ophthalmological sequelae upon initial infection, long-term effects remain to be seen. Patients may require long-term monitoring for inflammatory conditions that have not been identified in the acute setting of the disease.

Potential routes of viral transmission continue to be a cause of concern for both patients and providers alike. Well-studied routes of transmission include respiratory droplets and indirect contact. A study performed in South Korea showed that surfaces contaminated with Middle East respiratory syndrome coronavirus (MERS-CoV) tested positive via polymerase chain reaction (PCR) even 5 days after contact with a virus-positive patient. ${ }^{8}$ Current data remain unclear as to the prevalence of coronavirus-positive PCR within ocular secretions and the rate of ocular transmission 
in documented patients with COVID-19. Xia et al. reported a case series of 30 patients who were confirmed positive for coronavirus in which conjunctival secretions were collected for the reverse-transcription PCR (RT-PCR) assay. ${ }^{9}$ Results demonstrated that the tears and ocular secretions of coronarvirus-positive patients showing symptoms of conjunctivitis were also PCR-assay-positive. Conversely, the study confirmed that patients with COVID-19 without findings of conjunctivitis did not show evidence of viral shedding within their ocular secretions. ${ }^{9}$ Seah and Agrawal discussed the potential mechanism of ocular involvement, including routes such as direct inoculation via aerosolized droplets, hematogenous spread via the lacrimal gland, and migration from the nasopharynx. ${ }^{7}$ Due to the unclear rate of ocular transmission, strict adherence to safe practice guidelines remains essential.

\section{Clinical and surgical practice management}

While some degree of human contact is inevitable, there are many precautionary measures that clinics can take to reduce the risk of viral transmission. First, triaging for symptoms of COVID-19 prior to the visit is critical and should be applied to every patient in anticipation of their arrival. ${ }^{10-12}$ Body-temperature measurement has also been recommended as an objective screening tool in addition to patient history. ${ }^{13}$ Entrances to the clinic must be minimized, and the triage of patients should take place prior to their entry. During the triage process, patients should be asked about contacts with people who have tested positive for COVID-19, recent travel outside the country, and symptoms including fever, dry cough, dyspnea, sore throat, runny nose, myalgia, fatigue, headache, diarrhea, loss of smell, loss of taste, and nausea. ${ }^{14-17}$ Some practices have additionally reduced the number of in-clinic visits with adherence to strict timelines in order to prevent multiple patients accumulating in the waiting room. ${ }^{11}$

\section{Personal protective equipment}

Ophthalmic professionals come in contact with many patients each day, and their effort toward reducing the spread of the virus remains essential. Surgical facemasks should be mandatory for physicians, staff members, and patients in all ophthalmologic clinics. ${ }^{18}$ Frequent hand washing with alcohol-based hand rubs or with soap and water for at least 20 seconds should also be practiced. ${ }^{1}$ Furthermore, full personal protective equipment (PPE), including caps, gowns, N95 respirators, and eye goggles, should be worn when seeing patients with confirmed COVID-19 or persons under investigation (PUIS), or performing any surgical procedure. ${ }^{18}$ Protective breath shields attached to slit lamps have also been shown to offer some preventative benefit. ${ }^{19}$ Various slit lamp companies carry such attachments-or alternatively, comprehensive instructions can be found online enabling providers to make their own shields. ${ }^{19}$ In addition to standard PPE, many ophthalmologists regularly perform aerosolizing procedures, which may warrant a higher level of protection than merely droplet precaution. ${ }^{20,21}$ Droplets are classically defined as aqueous bodies larger than 5 microns and are a common mode for transmission of viruses. Aerosols, by contrast, are much smaller and can travel on air currents for several hours, causing them to be far more infectious.

\section{Ophthalmic instrument and environment disinfection}

Many ophthalmic instruments either come in close proximity to, or directly contact, the eye. Instruments that directly contact the patients' conjunctival mucosal surface include the eyelid speculum, tonometers, and ultrasound probes. Instruments that come in close proximity to the patient include optical coherence tomography scanners, slit-lamps, direct ophthalmoscopes, fundus cameras, and corneal topographers. Disinfection of these instruments can be achieved with 75\% ethanol or hydrogen peroxide immersion, or wipes. ${ }^{22,23}$ Any device that comes in contact with mucosal membranes should be disinfected after every use, while the rest should be disinfected at least daily. ${ }^{22,23}$ Routine thorough cleaning of waiting rooms and areas where patients gathering is advised. Attention should also be paid to the proper ventilation of patient areas with adequate air filtration and turnover directed at minimizing respiratory transmission. ${ }^{24}$

As a last measure, telehealth should be considered whenever feasible and reasonable. Many ophthalmology practices have had great success implementing virtual visits and using telehealth devices and smartphone applications easily accessible to patients. ${ }^{25-29}$ Despite recent advances, this technology has proven to be of limited benefit in the older population, particularly those who suffer from poor vision. ${ }^{26}$

\section{Treatment recommendations}

Currently, there are no compelling studies showing that COVID-19associated conjunctivitis should be treated differently than the most common type of adenoviral conjunctivitis. Standard treatments are supportive, addressing patient comfort measures with lubricants and cold compresses. The vast majority of cases will resolve without any treatment, although in refractory circumstances, topical antibiotics can be considered to prevent secondary bacterial infection. Certain antivirals, such as umifenovir, lopinavir, and ritonavir, have been investigated during the pandemic but show no clear benefit in reducing ocular symptoms..$^{30} \mathrm{As}$ with anyone experiencing symptoms due to COVID-19, the patient should be asked to quarantine prior to arranging a visit with their ophthalmologist. Telephone screening can be a useful tool for differentiating between an urgent and a non-urgent issue.

\section{Conclusions}

From an ophthalmology perspective, the disease burden of COVID-19 appears to be mild in comparison with other organ systems. Relatively few patients who test positive for COVID-19 demonstrate ocular symptoms, and in such cases the manifestations have not, thus far, been visually significant. Conjunctivitis remains the primary finding associated with infection, but in rare cases symptoms may include hyperemia, chemosis, and increased tearing. Current data are not conclusive in determining levels of infectivity and rates of transmission via ocular secretions. Due to the uncertain nature of ocular transmission, strict adherence to safety guidelines should be followed in all circumstances. Safe practice guidelines include proper donning of PPE, proper cleaning of ophthalmic equipment, and thorough screening and triage prior to appointments. Treatment of ocular symptoms related to COVID-19 has mostly been supportive in nature, trying to address patient comfort. Few antivirals and antibiotics have been investigated without clear benefit in relieving the most frequent manifestation of conjunctivitis. Safety guidelines pertaining to clinical practice continue to evolve as practitioners adapt to the new normal of providing healthcare under the burden of a global pandemic. With continued efforts focusing on reduction of transmission, we strive toward the goal of offering safe, quality, ophthalmological care. $\square$ 
1. Centers for Disease Control and Prevention. Infection control: Severe acute respiratory syndrome coronavirus 2 (SARS-CoV-2). 2020. Available at: www.cdc.gov/coronavirus/2019-ncov/ hcp/infection-control-recommendations.html?CDC_AA refVal=https\%3A\%2F\%2Fwww.cdc.gov\%2Fcoronavirus\%2F2019ncov\%2Finfection-control\%2Fcontrol-recommendations.html (accessed November 4, 2020)

2. Seah I, Su X, Lingam G. Revisiting the dangers of the coronavirus in the ophthalmology practice. Eye (Lond). 2020;34:1155-7.

3. KUO IC, O'Brien TP. COVID-19 and ophthalmology: an underappreciated occupational hazard. Infect Control Hosp Epidemiol. 2020;41:1207-8

4. Wu P, Duan F, Luo C, et al. Characteristics of ocular findings of patients with coronavirus disease 2019 (COVID-19) in Hubei Province, China. JAMA Ophthalmol. 2020:138:575-8.

5. Ozturker ZK. Conjunctivitis as sole symptom of COVID-19. A case report and review of literature. Eur $J$ Ophthalmol. 2020:1120672120946287. doi: 10.1177/1120672120946287. [Erratum: 2020: Aug 27:1120672120956486. doi: $10.1177 / 1120672120956486$.

6. Hu K, Patel J, Patel BC. Ophthalmic manifestations of coronavirus. 2020. Available at: www.ncbi.nlm.nih.gov/books/NBK556093/ (accessed January 14, 2021)

7. Seah I, Agrawal R. Can the coronavirus disease 2019 (COVID-19) affect the eyes? A review of coronaviruses and ocular implications in humans and animals. Ocul Immunol Inflamm. 2020;28:391-5

8. Bin SY, Heo JY, Song MS, et al. Environmental contamination and viral shedding in MERS patients during MERS-CoV outbreak in South Korea. Clin Infect Dis. 2016;62:755-60. [Errata: 2016;62:1328 and 2016;63:851.]

9. Xia J, Tong J, Liu M, et al. Evaluation of coronavirus in tears and conjunctival secretions of patients with SARS-COV-2 infection. J Med Virol. 2020;92:589-94.

10. Borrelli E, Sacconi R, Querques $L$, et al. Taking the right measures to control COVID-19 in ophthalmology: the experience of a tertiary eye care referral center in Italy. Eye (Lond). 2020;34:1175-6.

11. Lai THT, Tang EWH, Chau SKY, et al. Stepping up infection control measures in ophthalmology during the novel coronavirus outbreak: an experience from Hong Kong. Graefes Arch Clin Exp Ophthalmol. 2020;258:1049-55

12. Yu AY, Tu R, Shao $X$, et al. A comprehensive Chinese experience against SARS-CoV-2 in ophthalmology. Eye Vis (Lond). 2020;7:19.

13. Romano MR, Montericcio A, Montalbano C, et al. Facing COVID-19 in ophthalmology department Curr Eye Res. 2020;45:653-8.

14. Cao M, Zhang D, Wang $Y$, et al. Clinical features of patients infected with the 2019 Novel Coronavirus (COVID-19) in Shanghai, China. medRxiv. 2020;2020.03.0420030395. doi: 10.1101/2020.03.04.20030395

15. Hopkins C, Surda P, Kumar N. Presentation of new onset anosmia during the COVID-19 pandemic. Rhinology. 2020;58:295-8.

16. Mao $\mathrm{L}$, Jin $\mathrm{H}$, Wang $\mathrm{M}$, et al. Neurologic manifestations of hospitalized patients with coronavirus disease 2019 in Wuhan, China. JAMA Neurol. 2020;77:683-90.

17. Walker A, Hopkins C, Surda P. Use of Google Trends to investigate loss-of-smell-related searches during the COVID-19 outbreak. Int Forum Allergy Rhinol. 2020;10:839-47.

18. Gharebaghi R, Desuatels J, Moshirfar M, et al. COVID-19: Preliminary clinical guidelines for ophthalmology practices. Med Hypothesis Discov Innov Ophthalmol. 2020;9:149-58.

19. Wan KH, Huang SS, Young AL, et al. Precautionary measures needed for ophthalmologists during pandemic of the coronavirus disease 2019 (COVID-19). Acta Ophthalmol. 2020;98:221-2.
20. Koshy ZR, Dickie D. Aerosol generation from high-speed ophthalmic instrumentation and the risk of contamination from SARS COVID19. Eye (Lond). 2020;34:1954-5.

21. Wong $R$, Bannerjee $P$, Kumaran N. Aerosol generating procedures in intraocular surgery. Eye (Lond). May 28 2020;doi:10.1038/ S41433-020-0997-7.

22. Chan WM, Liu DT, Chan PK, et al. Precautions in ophthalmic practice in a hospital with a major acute SARS outbreak: an experience from Hong Kong. Eye (Lond). 2006;20:283-9.

23. Rutala WA. APIC guideline for selection and use of disinfectants 1994, 1995, and 1996 APIC Guidelines Committee. Association for Professionals in Infection Control and Epidemiology, Inc Am J Infect Control. 1996;24:313-42.

24. Nazarenko Y. Air filtration and SARS-COV-2. Epidemiol Health. 2020;42:e2020049.

25. Ansell K, G. Maconachie G, Bjerre A. Does the Eyechart app for iPhones give comparable measurements to traditional visual acuity charts? Br Ir Orthopt J. 2020;16:19-24.

26. Fatehi F, Jahedi F, Tay-Kearney M-L, et al. Teleophthalmology for the elderly population: A review of the literature. Int I Med Inform 2020;136:104089.

27. Ghazala FR, Hamilton R, Giardini MR, et al. Teleophthalmology techniques increase ophthalmic examination distance. Eye (Lond). 2020:1-2.

28. Kalavar M, Hua HU, Sridhar J. Teleophthalmology: an essential too in the era of the novel coronavirus 2019. Curr Opin Ophthalmol. 2020;31:366-73.

29. Saleem SM, Pasquale LR, Sidoti PA, et al. Virtual ophthalmology: Telemedicine in a COVID-19 era. Am J Ophthalmol. 2020;216:237-42.

30. Tsai DC, Huang YH, Chen SJ. Parafoveal atrophy after human amniotic membrane graft for macular hole in patients with high myopia. Br J Ophthalmol. 2020; doi: 10.1136/bjophthalmol-2019-315603. 